\title{
Hand tremor possibly induced by interaction of risperidone and lithium in a case of bipolar disorder with schizophrenia: a case report
}

\author{
Santanu Munshi ${ }^{1}$, Ranjita Santra (Dhali) ${ }^{1}{ }^{*}$, Indranil Saha ${ }^{2}$
}

\author{
${ }^{1}$ Department of Clinical and \\ Experimental Pharmacology, \\ Calcutta School of Tropical \\ Medicine, Kolkata, India \\ ${ }^{2}$ Department of Psychiatry, \\ Institute of Psychiatry Kolkata, \\ India
}

Received: 30 May 2016

Accepted: 01 July 2016

\section{*Correspondence to:}

Dr. Ranjita Santra (Dhali),

Email: dsdrranjita@gmail.com

Copyright: $\odot$ the author(s), publisher and licensee Medip Academy. This is an openaccess article distributed under the terms of the Creative Commons Attribution NonCommercial License, which permits unrestricted noncommercial use, distribution, and reproduction in any medium, provided the original work is properly cited.

\begin{abstract}
Drug-induced movement disorders possess a varied presentation and pose a challenging task for the physicians to diagnose such cases. A middle-aged woman with bipolar disorder comorbid with schizophrenia was under treatment with lithium carbonate, risperidone, sodium valproate, trihexiphenidyl tablets on a daily basis for two years. She experienced bilateral tremors of the hands which were present at rest and exacerbated by mental activity, when under observation and during voluntarily initiated activity. There were no associated extrapyramidal or cerebellar signs. The tremors persisted after withdrawal of sodium valproate and trihexiphenidyl, but disappeared completely after risperidone was stopped but lithium carbonate intake continued in an uninterrupted manner. Investigations did not reveal any positive finding. Hence, drug interaction may possibly be the offender for tremors in this case.
\end{abstract}

Keywords: Drug induced tremors, Lithium, Risperidone, Drug interaction, Bipolar disorder

\section{INTRODUCTION}

Lithium salts have been used in the prophylaxis and treatment of depression and bipolar disorder for more than 50 years. Lithium is a naturally occurring alkali metal and monovalent action chemically similar to $\mathrm{Na}+$ and $\mathrm{K}+$. The exact mechanism by which it stabilizes mood is not known. Medication-induced postural tremor is characterized by a regular, rhythmic oscillation of hands and fingers, head, mouth, or tongue. Tremor is usually classified according to the behaviour in which it occurs. ${ }^{1}$ Abnormal involuntary movements may appear in any part of the body, and can be embarrassing, distressing, disfiguring or dangerous. They commonly appear in the form of tremor, dystonia, or dyskinesia. Tremor is an involuntary rhythmic oscillation of one or parts of body about a fixed plane in space. ${ }^{2}$ Tremors are observed with the use of various drugs, including tricyclic antidepressants, monoaminoxidase inhibitors, antipsychotics, lithium, valproate, lamotrigine, antihistamines, thyroxine and nicotine. Drug-induced tremors are usually bilateral, action tremors. ${ }^{3}$

\section{CASE REPORT}

A 55 year old woman, with 2 years history of bipolar disorder comorbid with schizophrenia attended the psychiatry OPD with the chief complaints of bilateral tremors of both the hands for last seven days. On clinical examination, it was observed that there were regular rhythmic movements of her of both the hands, with no other extrapyramidal symptoms. The tremors increased 
with mental tasks, and especially in outstretched hands held against gravity. The tremors were course, rhythmic, regular in nature and present at the resting condition. The tremor used to disappear as the target was approached, thus did not cause any difficulty in carrying out her daily activities. The tremors disappeared during sleep. At the time of evaluation, detailed clinical examination including bedside neurological examination revealed no abnormalities. There was no history of any addiction substance abuse. The patient had been successfully maintained with lithium carbonate tablet (600 mg daily) for the past 2 years for her bipolar disorder. The patient was also prescribed sodium valproate tablets $500 \mathrm{mg}$ twice daily for bipolar disorder, metformin tablets 500 mg twice daily for type 2 diabetes and amlodipine tablets $5 \mathrm{mg}$ once daily for hypertension as evident from her treatment history.

One month ago, the dose of lithium carbonate was increased from $600 \mathrm{mg}$ daily to $900 \mathrm{mg}$ daily. Estimated serum lithium concentration was $0.71 \mathrm{mEq} / \mathrm{L}$ for $600 \mathrm{mg}$ daily dose of lithium and that measured at two weeks after increasing the dose to $900 \mathrm{mg}$ was $1.0 \mathrm{mEq} / \mathrm{L}$. Due to worsening of her symptoms, risperidone tablets $2 \mathrm{mg}$ twice daily and trihexiphenidyl tablets $2 \mathrm{mg}$ twice daily were added to her regimen.

After two weeks of starting risperidone tablets, the patient experienced tremors in both the hands sparing other parts of the body in spite of addition of trihexiphenidyl to her regimen. Serum Lithium estimation at that point of time revealed the level as $1.5 \mathrm{mEq} / \mathrm{L}$. The liver function tests showed raised liver enzyme SGOT which was $93 \mathrm{IU}$ in the first week which and then returned to the baseline value subsequently. Blood for plasma glucose revealed fasting value as $103 \mathrm{mg} / \mathrm{dl}$ and post-prandial value as $130 \mathrm{mg} / \mathrm{dl}$, thereby excluding the possibility of hypoglycemia as one of the causative factor for her tremors. Investigations for complete blood count, BUN, creatinine, electrolytes level, Wilson's disease, thyroid abnormalities and folic acid levels were normal. Tests for HIV 1 and 2, VDRL were negative. MRI brain and EMG were not performed as we suspected DIT in this case. Tablets sodium valproate and trihexiphenidyl were withdrawn for a week. After one week of their discontinuation, the tremors still persisted. Then, tablet risperidone was withdrawn for a week, thereafter leaving behind only lithium (900 mg daily) to be continued in addition to metformin and amlodipine as before. The patient was instructed to come to the Psychiatry OPD at two weeks after discontinuation of risperidone tablets. After two weeks of withdrawl of risperidone, the resting tremors subsided completely as was evident from the patient's history and clinical examination.

Tablet risperidone was substituted by tablet olanzapine (10 mg/day) to take for proper control of her disease symptoms, with re-start of sodium valproate and trihexiphenidyl in the same doses as before the de- challenge. Tablet metformin and amlodipine intake was in an uninterrupted manner.

Thus, the sequence of events in this case has helped us in establishing our suspicion of DIT. The drug interaction probability scale (DIPS) was used to evaluate drug interaction causation in our patient. The results showed a possible causal relationship (score of 4) between risperidone and lithium. Chronic toxicity happens in patients on chronic lithium therapy and the severity is categorized as mild (1.5 to $2.0 \mathrm{mEq} / \mathrm{L})$, moderate $(2.0$ to $2.5 \mathrm{mEq} / \mathrm{L}$ ) or severe (greater than $2.5 \mathrm{mEq} / \mathrm{L}$ ) based on the serum lithium concentration. But, in our case serum lithium concentration reached near to the lower limit of the range and can be said to be mild in nature.

\section{DISCUSSION}

Lithium and antipsychotic combination has been used to treat the bipolar disorder, although this combination has the potential to cause reversible to irreversible neurotoxicity. As evident from our case, serum lithium concentration accentuated from $1.0 \mathrm{mEq} / \mathrm{L}$ to $1.5 \mathrm{mEq} / \mathrm{L}$ after addition of the antipsychotic drug risperidone, thereby directing the clinicians to suspect a possible correlation in the pharmacokinetic behavior of such concomitant medications. The exact mechanism is unknown, but it has been postulated that this combination may cause toxicity by increasing dopamine receptor blockade or by changing lithium pharmacokinetic resulting in increasing intracellular level. ${ }^{4}$ The tremor induced by lithium is typically $8-12 \mathrm{~Hz}$, thereby falling into the category of enhanced physiological tremor, and mainly affects the hands. ${ }^{5}$ Tremor can occur over a wide range of lithium concentrations, with a correlation to lithium dose possible in some patients.

Concomitant therapy with certain drugs, such as antidepressants (tricyclics or SSRIs) or valproic acid, can potentiate the tremorogenic features of lithium. ${ }^{6}$ In this case, the tremors disappeared on reducing the dose of lithium and discontinuation of risperidone without tailoring the doses of trihexiphenidyl and valproic acid. One review estimated that about $27 \%$ of patients treated with lithium develop tremors, with individual studies showing wide variability from $4 \%$ to $65 \% .^{7}$ There is a suggestion that bipolar illness itself may increase the risk for antipsychotic-induced movement disorder. ${ }^{8}$ No interactions of lithium with metformin or amlodipine are found on literature search that could precipitate the tremors. The clinical examination and laboratory investigations ruled out any other causes of hand tremor and suggested a possibility of drug-induced tremor in this case.

\section{CONCLUSION}

Drug-induced tremor is an important clinical problem experienced by many patients of bipolar disorder. Treating clinicians can overlook pre-existing tremor 
before patients begin taking a tremorogenic drug. Clinicians should be aware of the potential drug-drug interactions while prescribing mood stabilizers with avoidance of the combinations as far as possible.

Funding: No funding sources

Conflict of interest: None declared

Ethical approval: The study was approved by the

Institutional Ethics Committee

\section{REFERENCES}

1. Sethi KD. Tremor. Curr Opin Neurol. 2003;16:481-5.

2. Anouti A, Koller WC. Tremor disorders: diagnosis and management. West J Med. 1995;162:510-3.

3. Bain P. The management of tremor. J Neurol Neurosurg Psychiatry. 2002;72(Suppl 1):i3-9.
4. Boora K, Xu J, Hyatt J. Encephalopathy with combined lithium risperidone administration. Acta Psychiatr Scand. 2008;117:394-6.

5. Factor SA. Lithium-induced movement disorders. In: Sethi KD, ed. Drug-induced movement disorders. New York: Marcel Dekker; 2004:209-31.

6. Zaninelli R, Bauer M, Jobert M, MullerOerlinghausen B. Changes in quantitatively assessed tremor during treatment of major depression with lithium augmented by paroxetine and amitriptyline. J Clin Psychopharmacol. 2001;21:190-8.

7. Gelenberg AJ, Jefferson JW. Lithium tremor. J Clin Psychiatry. 1995;56:283-7.

8. Van Rossum I, Tenback D, van Os J. Bipolar disorder and dopamine dysfunction: an indirect approach focusing on tardive movement syndromes in a naturalistic setting. BMC Psychiatry. 2009;9:16.

Cite this article as: Munshi S, Santra R, Saha I. Hand tremor possibly induced by interaction of risperidone and lithium in a case of bipolar disorder with schizophrenia: a case report. Int J Basic Clin Pharmacol 2016;5:1684-6. 